\title{
BODIPY dimers: structure, interaction, and absorption spectrum
}

\author{
Patryk Rybczynski ${ }^{1}$ · Anna Kaczmarek-Kędziera ${ }^{1}$ (D)
}

Received: 23 December 2020 / Accepted: 24 February 2021 / Published online: 26 March 2021

(C) The Author(s) 2021

\begin{abstract}
The object of the present study are BODIPY molecules obtained previously by Piskorz et al. (Dyes Pigm. 178:108322, 2020) for their antimicrobial activity. Structural analysis of the BODIPY dimers is presented in context of the aggregation influence on the photophysical properties. The thorough investigation of the nature of intermolecular interaction in the representative BODIPY dimers is provided together with the decomposition of the interaction energy into the components of well-defined origin according to SAPT procedure. For the model BODIPY systems the careful examination of the interaction nature for the dimer structure based on experimental crystal study as well as fully optimized is given. The tendencies observed in the model dimers are further on investigated for two pairs of BODIPY systems designed for biomedical application. The analyzed molecules are shown to maximize the mutual interaction by the optimization of the stacking dispersion contacts between the aromatic rings of the molecules, therefore producing stable dimers. The estimation of SAPT0 interaction energy components confirms the dominating dispersion character arising from mutual BODIPY core contacts. The influence of the dimerization process on the photophysical properties of the systems studied theoretically depends to the high extend on the dimerization mode and is significant for parallel and antiparallel dispersion-governed dimers.
\end{abstract}

Keywords BODIPY $\cdot$ Density functional calculations $\cdot$ Interaction energy $\cdot$ Aggregation $\cdot$ Electronic spectra

\section{Introduction}

4,4-Difluoro-4-bora-3a,4a-diaza-s-indacene derivatives, commonly known as BODIPY dyes, belong to the class of organic systems bearing the $\mathrm{BF}_{2}$ moiety connecting two five-membered heterocyclic pyrrole rings. They are known for over 50 years and still gain much attention due to their particular photooptical properties, namely strong absorption of the visible light and significant fluorescence quantum yield [1,2]. The practical applications of BODIPYs can be limited to some extend due to their poor absorption above $600 \mathrm{~nm}$ or weakly populated triplet states. However, their relatively simple chemical modification allows for precise tailoring of their emission or absorption features for instance by controlled shifting of the absorption or fluorescence range or promotion of the intersystem crossing

Anna Kaczmarek-Kędziera

teoadk@umk.pl

Patryk Rybczynski

pat_ryb@doktorant.umk.pl

1 Faculty of Chemistry, Nicolaus Copernicus University in Torun, Gagarina 7, 87-100 Torun, Poland
[3-5]. This further on permits to obtain systems exhibiting strong phosphorescence, significant singlet oxygen generation quantum yield or absorbing red light for the potential biomedical applications.

BODIPYs show good stability both to environmental changes (such as $\mathrm{pH}$ [6]) and light [7]. Yet, due to the nonpolar nature of BODIPY core, they tend to aggregate in highly polar solvents [8]. The intermolecular interactions are known to strongly affect the photophysical properties of different fluorophores by the undesired effect of fluorescence quenching due to the $\pi-\pi$ stacking among others [9-11]. The peculiar behavior of BODIPY probes has been also reported in water [12], what can restrict the utilization of these dyes in fluorescence labeling or sensing in biomedical environment. Therefore, one can expect that the obnoxious aggregation or interaction with solvent molecules can deteriorate the favorable BODIPY features. The controlled and well-defined aggregation of the fluorophores can on the other hand provide very profitable way of modulation of their photophysical properties for given applications.

One of the first complex investigations of the influence of BODIPY interactions in dimers on their electrooptical properties has been presented by Bergström [13]. Two BODIPY molecules were covalently linked to the protein plasminogen 
activator inhibitor-1 (PAI-1) at the positions of the mutant S344C and M347C residues. The recorded spectra in protein and in lipid phase has provided the confirmation of the two types of dimers present in the systems. In protein, nonemissive dimers are formed, for which the intensive absorption is registered at $477 \mathrm{~nm}$. The stacking dimer with parallel dipole moments is thus shown to cause the wavelength blueshift with respect to the monomer spectrum (absorption maximum at $505 \mathrm{~nm}$ ). On the other hand, in lipids the low energy modes are present, exhibiting absorption at $570 \mathrm{~nm}$ and broad emission at $630 \mathrm{~nm}$. These dimers are ascribed to the antiparallel arrangement of the BODIPY electronic transition dipole moments. These findings open the possible application of BODIPY dimers in investigation of protein structure and folding.

The solid-state aggregation-induced emission of BODIPY dyes has been found for the meso- $\mathrm{CF}_{3}$-substituted systems [14]. In comparison to the meso-methyl-substituted BODIPY, the introduction of strongly electron withdrawing group has lead to the formation of highly emissive J-aggregates characterized in a coplanar inclined arrangement according to the requirements of the Kasha's exciton model. The relatively simple substitution analyzed by Choi et al. and the definite microstructural determination by $\mathrm{X}$ ray diffraction study allows developing the beneficial ways of constructing the optoelectronic solid-state devices [14].

It has also been shown that the tendency of the BODIPY dye to self-assembly in the aquatic environment can be applied as the tool for the improvement of the anticancer drug activity. Capsaicin, used in the prostate cancer therapy, attached covalently to the hydrophobic BODIPY core, allows avoiding the necessity of the drug encapsulation in nanocarriers and thus, decrease the administered doses and increase the accumulation in target tissues [15].

BODIPY dimer formation remains of interest nowadays when the new covalent or non-covalent ways of controlling the intramolecular arrangements in BODIPY aggregates is desired for instance for detection of analytes in aqueous biological environments [8, 16]. The explanation of the modulation of the photophysical properties of BODIPY can be provided on the basis of the exciton model proposed by Kasha [8, 17, 18]. The proper substitution of the dihydronaphthalene fused BODIPY cores leads to the concentration-dependent excimer-type NIR fluorescence. Another proposed approach relies on the host-guest type of complexes involving metal-organic cages for reducing the structural flexibility of the BODIPY dimers confined in the cavity. It has been shown that depending on the substitution of the BODIPY molecule, they tend to form either antiparallel $\mathrm{H}$-aggregates with blue-shifted absorption or the parallel J-aggregates with decreased excitation energy with respect to the monomeric species. However, the polycationic cages due to the limited space inside the cavity force even the J-preferring BODIPY dyes to form the $\mathrm{H}$ aggregates. This phenomenon opens the new possibilities of reversible molecular switching between the different type of arrangements of BODIPY dyes, leading to the controlled tunability of their absorption and emission [16]. The significance of these findings together with the general lack of control upon modification of the aggregation mode invokes the strong necessity of the thorough knowledge of the nature of intermolecular interaction in BODIPY dimers and-further on-higher aggregates. However, up to date no comprehensive study of these aspects has been provided. Only the scarce reports are available, considering the decomposition of the total interaction energy in BODIPY dyes into the physically meaningful components [19].

The present study is devoted to the new BODIPY derivatives, synthesized recently for the photodeactivation of bacteria [20]. The BODIPY core has been substituted in mesoposition by aromatic ring with terminally attached $\mathrm{N}$-alkyl phthalimide moiety of known biomedical activity, for instance the strong antimicrobial effect (see Fig. 1). For comparison also the analog with the terminal amino group has been obtained and investigated. Moreover, the substitution in 2 and 6 position by heavy atom has been introduced in order to increase the intersystem crossing to the triplet states. The analyzed BODIPY compounds have been shown to exhibit the red-shifted absorption range, highly desirable for biomedical applications, and strong singlet oxygen generation yield for iodinated species. The photophysical properties and photostability of these compounds have been carefully investigated in our group and will be published elsewhere [Rybczynski P, Smolarkiewicz-Wyczachowski A, Ziegler-Borowska M, Bocian S, Piskorz J, KaczmarekKedziera A, Photochemical properties and stability of BODIPY dyes, in preparation]. Here, the investigation of the intermolecular interaction in different types of dimers for the above mentioned BODIPY dyes as well as for the model compound $\mathbf{3}$ with well-defined crystal structure is provided. Additionally, the influence of the dimer formation on the absorption spectra of six BODIPY systems is discussed.

\section{Methodology}

Two pairs of BODIPY dyes are investigated with respect to the nature of intermolecular interactions in self-aggregates: the systems denoted as $\mathbf{1}$ contain the meso-substituent ended with the phthalimide moiety, while the $\mathbf{2}$ systems are terminated in that place by a small amino group. One system in each pair is iodinated in the 2,6-positions of the $\mathrm{BF}_{2}$-bearing ring (denoted further on as $\mathbf{- I}$ ) and the other one unsubstituted (protons in 2 and 6 positions, -H). All four structures of the initial systems were synthesized and characterized experimentally and are described elsewhere 
Fig. 1 Investigated BODIPY molecules: a 1-H for R=H, 1-I for $\mathrm{R}=\mathrm{I}$, $\mathbf{b}$ 2-H for R=H, 2-I for $\mathrm{R}=\mathrm{I}$, $\mathbf{e}$ atom numbering for $\mathbf{3}-\mathbf{H}$ for $\mathrm{R}=\mathrm{H}, \mathbf{3}-\mathrm{I}$ for $\mathrm{R}=\mathrm{I}$

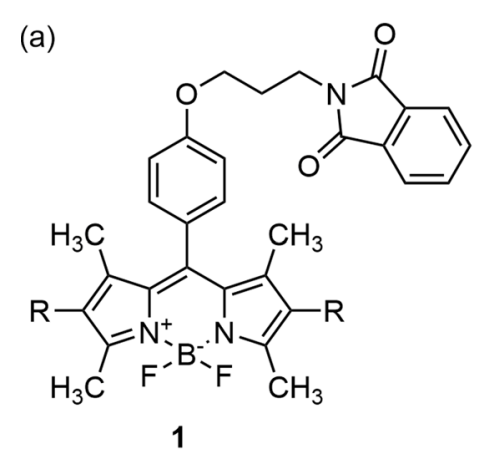

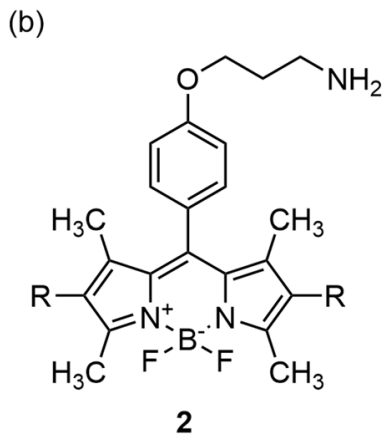

(c)

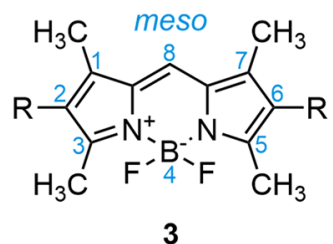

[20]. The monomer structures and properties are discussed together with the photostability of the investigated systems in context of their potential biomedical applications [Rybczynski P, Smolarkiewicz-Wyczachowski A, ZieglerBorowska M, Bocian S, Piskorz J, Kaczmarek-Kedziera A, Photochemical properties and stability of BODIPY dyes, in preparation]. Additionally, the two model BODIPY systems have been included in the present study, consisting of the simple 1,3,5,7-methyl-substituted core with no extended meso-substituent. For the consistency of the notation, these systems are denoted further on as $\mathbf{3}-\mathbf{I}$ and $\mathbf{3}-\mathbf{H}$, respectively, when iodinated or hydrogenated in the 2 and 6 positions.

The model systems $\mathbf{3 - H}$ and $\mathbf{3 - I}$ are investigated as the prototypical moieties for the careful analysis of the nature of the intermolecular interactions between the BODIPY cores in protonated and iodinated systems. The initial dimers of the four investigated dyes are constructed from two structures: first class of dimers arise from the lowest energy isomer which is the bent structure, where the mesosubstituent is directed to the BODIPY central ring, and the second class contains the dimers of the extended isomers characterized by the relative energy of monomers of about $8 \mathrm{kcal} / \mathrm{mol}$ for $\mathbf{1}$ and about $3 \mathrm{kcal} / \mathrm{mol}$ for 2 .

The full geometry optimization has been performed for several possible dimers of the four investigated structures with the $\omega \mathrm{B} 97 \mathrm{X}-\mathrm{D} / \mathrm{def} 2-\mathrm{SVP}$ approach in the gas phase. The choice of the improved def2- basis sets introduced by Weigend and Ahlrichs is rationalized by their flexibility, electric core potentials for heavy atoms, namely iodine, including small core for the proper treatment of scalar relativistic effects and their recommended application for DFT approaches [21-26]. The $\omega$ B97X-D functional on the other hand has been selected due to its wide range of applicability and confirmed controlled behavior for the ground state properties and non-covalent interactions [27].

All of the obtained dimers are presented in Figs. S1S4 of Supplementary Information. The character of the stationary points on the potential energy surface of all of the investigated dimers is confirmed by the harmonic vibrational analysis. The nature of the mutual interactions has been investigated with the SAPT0 decomposition scheme with aug-cc-pVDZ basis set ensuring the proper balance between the quality of the obtained data and the costs of the calculations. Due to the large size of the substituted dimers, in particular $\mathbf{1 - H}$ and $\mathbf{1 - I}$, the calculations will be also performed in def2-SVP basis set and their accuracy will be compared with the aug-cc-pVDZ basis set, recommended as providing best error cancellation [28-31]. The vertical excitation energies together with the corresponding frontier orbitals for the dimers have been determined within the M06-2X functional recommended as the most adequate for the description of the photophysical properties of the BODIPY dyes [32-39].

For the investigation of the solvent influence on the aggregation phenomena, also the geometry optimization has been carried out within the continuum solvent model for tetrahydrofuran, acetonitrile and water. However, since the dimer structures do not differ qualitatively with respect to the vacuum-optimized ones, the further discussion is concentrated only on vacuum calculations.

The impact of the mutual interaction on the photophysical properties of the investigated BODIPY dyes is analyzed within the TD-DFT approach. The one-photon absorption spectra are calculated in vacuum with several functionals recommended to the electronic absorption description: CAM-B3LYP, M06-2X and conventional hybrid PBE0 one. The Weigend def2-SVP basis set is applied.

\section{Dimer structures}

\section{Model dimers and crystal structure}

The crystallographic structure of model $\mathbf{3}-\mathbf{H}$ has been investigated [40]. The unit cell consists of four $\mathbf{3 - H}$ molecules, presented in Fig. 2, panel a. Panels b-d of Fig. 2 show three dimers in the geometry based on the crystal: first one with the parallel (significantly) displaced arrangement (panel b), and the two latter with the perpendicular structure, where fluorine atom of one molecule interacts directly with the BODIPY core hydrogen in position 2 and 6 (panel c) or methyl group in position 1 or 7 (panel $d$ ). The 


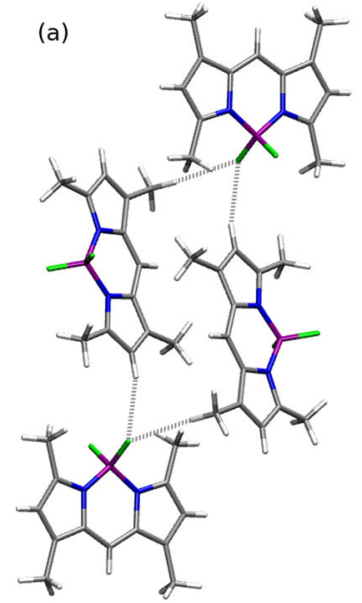

(b)

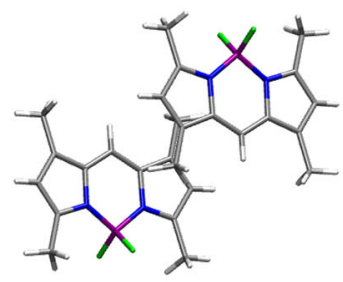

(c)

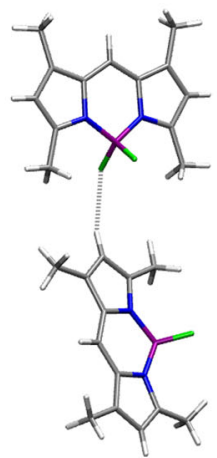

(d)

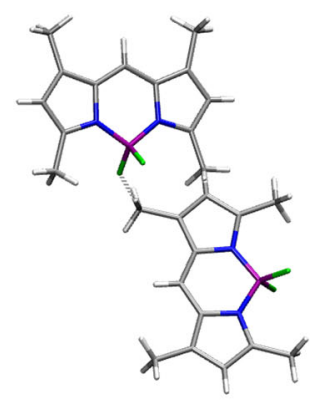

Fig. 2 Interaction in the crystal structure of 3-H: a four molecules in a unit cell, and dimers based on the experimentally determined crystal structure: b parallel displaced, c perpendicular

supermolecular counterpoise-corrected interaction energy, $\triangle E_{S M}^{C P}$, in dimers and in the tetramer from Fig. 2 and the SAPT0 total dimer interaction are summarized in Table 1. One can easily see that the most favorable interaction takes place in the parallel displaced dimers, even though the displacement leads in fact to almost no overlap of the aromatic part of the BODIPY scaffold. The distance between the closest atoms in the two molecules is observed between the hydrogen of the methyl group in position 1 and the nitrogen of the other molecule and amounts to $3.04 \AA$. The mutual supermolecular attraction in this type of dimer is of the order of $12 \mathrm{kcal} / \mathrm{mol}$ (see Table 1) and remains almost unchanged independently on the approach applied (except MP2/def2-SVP estimation). On the other hand the
$\mathrm{H}-\mathrm{F}$, d perpendicular $\mathrm{CH}_{3}-\mathrm{F}$ (the following atom colors are applied: carbon-gray, hydrogen-white, boron-magenta, nitrogen-blue, fluorine-green)

two remaining dimers, namely depicted in panels $\mathrm{c}$ and $\mathrm{d}$, are bound together via the weak $\mathrm{C}-\mathrm{H}$...F interaction and the corresponding $\triangle E_{S M}^{C P}$ does not exceed -3.85 and -4.58 $\mathrm{kcal} / \mathrm{mol}$, respectively.

The decomposition of the total SAPT0 interaction energy for the 3-H BODIPY dimers based on the experimentally determined crystal structure into the physically meaningful components is presented in Table 2. For all of the analyzed dimers the interaction is governed by the dispersion component, with the dispersion-to-electrostatic ratio equal respectively to $2.71,4.38$ and 1.77 for parallel, perpendicular $\mathrm{H}-\mathrm{F}$ and perpendicular $\mathrm{CH}_{3}-\mathrm{F}$ pairs. Table 2 is supplemented with the results of def2-SVP basis set for 3-H dimers for the sake of the comparison of the quality

Table 1 Counterpoise-corrected supermolecular interaction energy $\Delta E_{s m}^{C P}[\mathrm{kcal} / \mathrm{mol}]$ estimated for the 3-H dimers based on the experimentally determined crystal structure presented in Fig. 2 (panels a, b, c and d) within the $\omega$ B97X-D, MP2 and SAPT0 approach

\begin{tabular}{|c|c|c|c|c|c|}
\hline \multirow[b]{3}{*}{ System } & \multirow[b]{3}{*}{$\# \mathrm{bf}$} & \multicolumn{2}{|l|}{ Tetramer } & \multicolumn{2}{|l|}{ Dimers } \\
\hline & & Panel (a) & Panel (b) & Panel (c) & Panel (d) \\
\hline & & & Parallel & Perpendicular H-F & Perpendicular $\mathrm{CH}_{3}-\mathrm{F}$ \\
\hline \multicolumn{6}{|l|}{$\omega \mathbf{B 9 7 X - D}$} \\
\hline def2-SVP & 1308 & -28.57 & -12.64 & -3.62 & -4.50 \\
\hline def2-TZVP & 2592 & -29.19 & -12.60 & -3.85 & -4.58 \\
\hline def2-TZVPD & 3228 & & -12.58 & -3.84 & -4.56 \\
\hline \multicolumn{6}{|l|}{ MP2 } \\
\hline def2-SVP & 1308 & -18.67 & -8.53 & -2.18 & -2.93 \\
\hline def2-TZVP & 2592 & & -11.49 & -2.95 & -3.86 \\
\hline \multicolumn{6}{|l|}{ SAPT0 } \\
\hline aug-cc-pVDZ & 1098 & & -13.86 & -3.35 & -4.73 \\
\hline
\end{tabular}

\#bf denotes the number of basis functions for the tetramer 
Table 2 SAPT0 interaction energy components $[\mathrm{kcal} / \mathrm{mol}]$ for the 3-H dimer geometry based on the experimentally determined crystal structure

\begin{tabular}{|c|c|c|c|c|c|c|}
\hline \multirow[b]{2}{*}{ Component } & \multicolumn{3}{|c|}{ Aug-cc-pVDZ } & \multicolumn{3}{|c|}{ Def2-SVP } \\
\hline & Parallel & $\begin{array}{l}\text { Perpendicular } \\
\mathrm{H}-\mathrm{F}\end{array}$ & $\begin{array}{l}\text { Perpendicular } \\
\mathrm{CH}_{3}-\mathrm{F}\end{array}$ & $\begin{array}{l}\text { Parallel } \\
\text { H-F }\end{array}$ & $\begin{array}{l}\text { Perpendicular } \\
\mathrm{CH}_{3}-\mathrm{F}\end{array}$ & Perpendicular \\
\hline Electrostatic & -5.31 & -0.84 & -2.42 & -5.51 & -0.82 & -2.54 \\
\hline Exchange & 7.22 & 1.71 & 2.45 & 7.08 & 1.68 & 2.42 \\
\hline Induction & -1.36 & -0.54 & -0.49 & -1.25 & -0.49 & -0.46 \\
\hline Dispersion & -14.40 & -3.68 & -4.28 & -11.02 & -2.60 & -3.14 \\
\hline Total & -13.86 & -3.34 & -4.28 & -10.70 & -2.23 & -3.72 \\
\hline Disp/Elst & 2.71 & 4.38 & 1.77 & 2.00 & 3.16 & 1.24 \\
\hline
\end{tabular}

of the obtained data in order to shed the light on the larger system calculations. Despite the large difference of the number of basis set functions applied in aug-cc-pVDZ and def2-SVP for $\mathbf{3 - H}$, one can notice the qualitatively correct results gathered with the smaller basis set. From the practical point of view for the qualitative analysis performed here, the only shortcoming of the def-SVP basis set arises from the fact that the dispersion component is significantly underestimated with the smaller basis set (errors of the order of 25 to $30 \%$ ). This is however the expected consequence of the non-saturated diffuse part of the basis set, influencing the variational dispersion component, and as such, it allows the large system calculations to be performed with a reliable accuracy keeping in mind this issue. Therefore, for the BODIPY molecules with the large side substituent, particularly the phthalimide one (1-H, 1-I), the smaller basis set will be applied for SAPT0 calculations.

In order to compare the possibility of the BODIPY aggregation in crystal and in solution, also the investigation of the fully optimized dimers has been performed. The structure of model dimers of 3-I and 3-H optimized in vacuum are presented in Figs. 3 and 4. Corresponding structures in solvents (tetrahydrofuran, acetonitrile and methanol) does not differ much in principle and therefore they are omitted in further discussion. Four types of initial structures have been investigated: planar, stacked parallel, stacked antiparallel and perpendicular. Full geometry optimization has led to the five stable dimers of $\mathbf{3 - H}$, namely planar, perpendicular and three different stacked arrangements, while in the 3-I dimer the perpendicular structure with the iodine interacting with the fluorine in the $\mathrm{BF}_{2}$ moiety has been found. The relative energies of these structures suggest that the most favorable is the stacking type of interaction which brings the most stable antiparallel structure and the parallel dimer of the relative energy of $7.08 \mathrm{kcal} / \mathrm{mol}$ for $\mathbf{3}-\mathbf{I}$. The dimers that do not benefit from the side interaction, lie more than $20 \mathrm{kcal} / \mathrm{mol}$ higher in the energy range (planar 3-I: 22.59 $\mathrm{kcal} / \mathrm{mol}$ and perpendicular 3-I: $25.02 \mathrm{kcal} / \mathrm{mol})$. In the case of $\mathbf{3 - H}$, the stacked antiparalell dimer exhibits the lowest energy of all analyzed forms, while the stacked twisted arising from the parallel initial arrangement (see Fig. 3, panel b) appears by $3.71 \mathrm{kcal} / \mathrm{mol}$ higher on the energy scale. Similarly as in the case of the iodinated system, the planar 3-H dimer is $18.31 \mathrm{kcal} / \mathrm{mol}$ higher in the energy range than the most stable form.

Table 3 presents the interaction energy for the obtained 3-I and 3-H dimers. It confirms clearly that the most important attractive contribution appears in the stacked systems, independently on the stacking mode (parallel as for iodinated derivative in Fig. 4 panel c, antiparallel as panels d of Figs. 3 and 4 or twisted as in Fig. 3 panels $c$ and e or in Fig. 4 panel e). The comparison of the structural arrangement of these fully optimized dimers with the motifs based directly on the experimental crystal geometry (a)
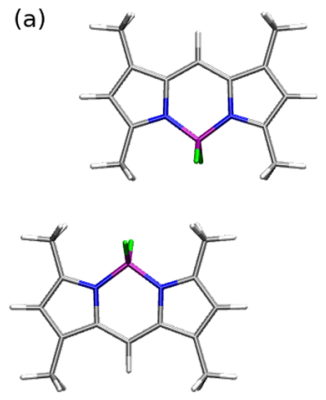

(b)
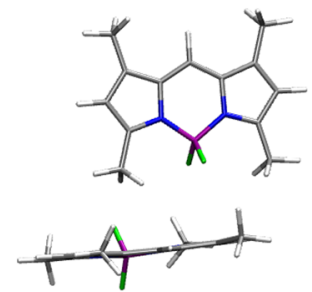

(c)

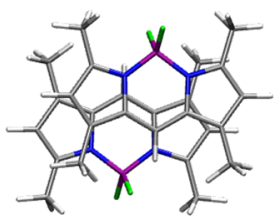

(d)

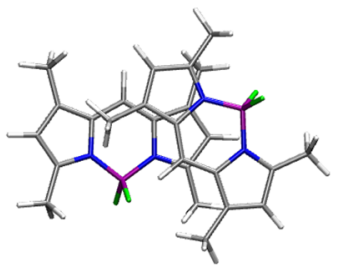

(e)

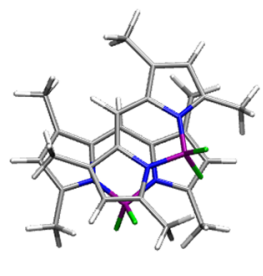

Fig. 3 Investigated optimized dimers of 3-H: a planar, b perpendicular, c-e stacked 
(a)

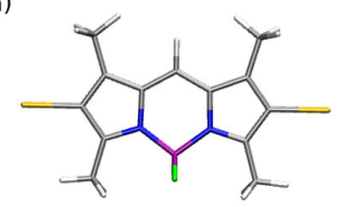

(b)
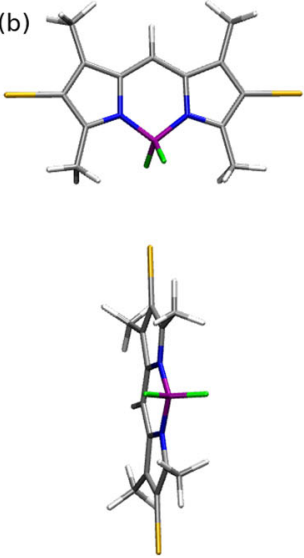

(c)

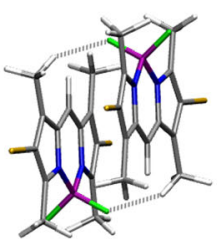

(d)

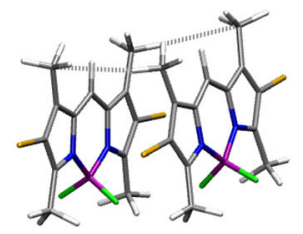

(e)

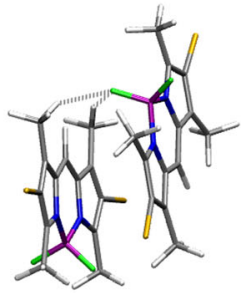

Fig. 4 Investigated optimized dimers of 3-I: a planar, b perpendicular, $\mathbf{c}-\mathbf{e}$ stacked

(compare Fig. 2) exhibits several intriguing issues. First of all, the full geometry relaxation in vacuum for $\mathbf{3 - H}$ does not lead to the stable perpendicular structure similar to those observed in crystal (Fig. 2, panel c). Such an arrangement is only found on the potential energy surface of 3-I. The absence of iodine on the other hand leads to the preferential perpendicular arrangement with the $\mathrm{BF}_{2}$ moiety of one molecule pointing to the pyrrolic ring of the other. Moreover, the presence of the iodine atoms lead to the additional stabilization of the stacked antiparallel and twisted structures with respect to the unsubstituted $\mathbf{3}-\mathbf{H}$ dimers. This effect can be ascribed to the particular balance between different SAPT0 components (Tables 4 and 5), since both electrostatic and dispersion part of interaction energy are more negative for 3-I together with the more positive exchange component than in $\mathbf{3 - H}$. On the other hand, in the case of the perpendicular arrangement, 3-H dimer becomes much stronger bonded than 3-I, however it also adopts different type of the relative position of the units and therefore cannot be directly related.
These observations for fully optimized arrangements are of importance from the point of view of intermolecular interactions in solution, which in turn can significantly affect the photophysical properties of investigated systems.

\section{meso-substituted BODIPY derivatives}

Among the fully optimized structures of 1-H, 1-I, 2-H and 2-I, discussed elsewhere [Rybczynski P, SmolarkiewiczWyczachowski A, Ziegler-Borowska M, Bocian S, Piskorz J, Kaczmarek-Kedziera A, Photochemical properties and stability of BODIPY dyes, in preparation], two general types have been chosen in order to construct the initial dimer structures: and elongated unfolded. This is of the most importance for the phthalimide-substituted BODIPY derivative, since the bent structure can benefit from the intramolecular stabilizing dispersion interaction between the BODIPY core and the aromatic ring in the terminal substituent. Such a bent structure limits to the high extend the possible mutual arrangement in the dimers and hinders

Table 3 Counterpoise-corrected supermolecular interaction energy $\Delta E_{s m}^{C P}[\mathrm{kcal} / \mathrm{mol}]$ estimated for the fully optimized dimers, presented in Figs. 3 and 4 (panels a to e), within the $\omega \mathrm{B} 97 \mathrm{X}-\mathrm{D} / \mathrm{def} 2-\mathrm{SVP}$ approach

\begin{tabular}{|c|c|c|c|c|c|c|}
\hline \multirow[b]{2}{*}{ System } & \multirow[b]{2}{*}{$\# \mathrm{bf}$} & \multirow{2}{*}{$\begin{array}{l}\text { Planar } \\
\text { Panel (a) }\end{array}$} & \multirow{2}{*}{$\begin{array}{l}\text { Perpendicular } \\
\text { Panel (b) }\end{array}$} & \multicolumn{3}{|l|}{ Stacked } \\
\hline & & & & Panel (c) & Panel (d) & Panel (e) \\
\hline \multicolumn{7}{|c|}{ def2-SVP } \\
\hline 3-I & 738 & -4.67 & -4.10 & -27.55 & -18.06 & -24.05 \\
\hline 3-H & 654 & -3.59 & -7.86 & -22.74 & -18.99 & -16.01 \\
\hline \multicolumn{7}{|c|}{ def2-TZVP } \\
\hline 3-I & 1472 & -5.45 & -4.24 & -27.48 & -18.54 & -24.37 \\
\hline 3-H & 1296 & -4.37 & -8.59 & -23.00 & -19.37 & -16.74 \\
\hline \multicolumn{7}{|c|}{ def2-TZVPD } \\
\hline 3-I & 1814 & -5.42 & -4.26 & -27.56 & -18.62 & -24.40 \\
\hline 3-H & 1614 & -4.35 & -8.59 & -23.06 & -19.38 & -16.78 \\
\hline
\end{tabular}


Table 4 SAPT0 interaction energy components [kcal/mol] estimated for the fully optimized 3-H dimers, presented in Fig. 3 (panels a to e), within the aug-cc-pVDZ and def2-SVP basis sets

\begin{tabular}{|c|c|c|c|c|c|}
\hline \multirow[b]{2}{*}{ System } & \multirow{2}{*}{$\begin{array}{l}\text { Planar } \\
\text { Panel (a) }\end{array}$} & \multirow{2}{*}{$\begin{array}{l}\text { Perpendicular } \\
\text { Panel (b) }\end{array}$} & \multicolumn{3}{|l|}{ Stacked } \\
\hline & & & $\begin{array}{l}\text { Anti } \\
\text { panel (c) }\end{array}$ & $\begin{array}{l}\text { Twisted1 } \\
\text { panel (d) }\end{array}$ & $\begin{array}{l}\text { Twisted2 } \\
\text { panel (e) }\end{array}$ \\
\hline $3-\mathbf{H}$ & aug-cc-pVDZ & & & & \\
\hline Electrostatic & -3.90 & -5.61 & -18.19 & -14.71 & -10.49 \\
\hline Exchange & 8.49 & 13.05 & 29.14 & 24.81 & 26.41 \\
\hline Induction & -4.12 & -5.69 & -8.44 & -7.93 & -8.17 \\
\hline Dispersion & -6.82 & -14.42 & -37.03 & -31.26 & -34.57 \\
\hline Total & -6.34 & -12.66 & -34.53 & -29.08 & -26.82 \\
\hline Disp/Elst & 1.75 & 2.57 & 2.04 & 2.13 & 3.30 \\
\hline $3-\mathbf{H}$ & def2-SVP & & & & \\
\hline Electrostatic & -3.85 & -5.61 & -20.03 & -15.87 & -11.06 \\
\hline Exchange & 7.91 & 13.26 & 33.30 & 27.57 & 29.46 \\
\hline Induction & -1.54 & -2.43 & -5.24 & -4.55 & -4.64 \\
\hline Dispersion & -4.67 & -10.63 & -28.95 & -24.25 & -26.77 \\
\hline Total & -2.15 & -5.41 & -20.92 & -17.11 & -13.01 \\
\hline Disp/Elst & 1.21 & 1.89 & 1.44 & 1.52 & 2.42 \\
\hline
\end{tabular}

the probability of the attraction of the two aromatic parts of each molecule.

Figure 5 presents the vacuum-optimized structures of the selected analyzed dimers for $\mathbf{1 - H}$ and $\mathbf{1 - I}$, and the corresponding structures of $\mathbf{2}-\mathbf{H}$ and $\mathbf{2}-\mathbf{I}$ are shown in Fig. 6. The supermolecular interaction energy and the total SAPT0 interaction energy are summarized in Table 6. Their values clearly show that the introduction of the iodine atom to the molecule increases the strength of mutual interactions except for the case of the 2-I unfolded dimer. This variation from the tendency can be ascribed to the different arrangement of the two molecules in the dimer for the iodinated and non-iodinated species. Namely, the unsubstituted system prefers the twisted orientation, while

Table 5 SAPT0 interaction energy components [kcal/mol] estimated for the fully optimized 3-I dimers, presented in Fig. 4 (panels a to e), within the aug-cc-pVDZ basis set for all atoms and def2-SVP for iodine or def2-SVP for all atoms

\begin{tabular}{|c|c|c|c|c|c|}
\hline \multirow[b]{2}{*}{ System } & \multirow{2}{*}{$\begin{array}{l}\text { Planar } \\
\text { Panel (a) }\end{array}$} & \multirow{2}{*}{$\begin{array}{l}\text { Perpendicular } \\
\text { Panel (b) }\end{array}$} & \multicolumn{3}{|l|}{ Stacked } \\
\hline & & & $\begin{array}{l}\text { Anti } \\
\text { panel (c) }\end{array}$ & $\begin{array}{l}\text { Twisted1 } \\
\text { panel (a) }\end{array}$ & $\begin{array}{l}\text { Twisted2 } \\
\text { panel (e) }\end{array}$ \\
\hline 3-I & \multicolumn{5}{|c|}{ aug-cc-pVDZ and for I: def2-SVP } \\
\hline Electrostatic & -4.71 & -5.33 & -22.12 & -12.09 & -18.31 \\
\hline Exchange & 8.36 & 7.69 & 40.01 & 29.39 & 32.68 \\
\hline Induction & -1.40 & -1.64 & -6.05 & -3.09 & -4.95 \\
\hline Dispersion & -8.02 & -5.56 & -46.89 & -38.90 & -39.99 \\
\hline Total & -5.75 & -4.84 & -35.05 & -24.68 & -30.58 \\
\hline Disp/Elst & 1.70 & 1.04 & 2.12 & 3.22 & 2.18 \\
\hline 3-I & \multicolumn{5}{|l|}{ def2-SVP } \\
\hline Electrostatic & -4.76 & -5.68 & -22.93 & -12.02 & -18.96 \\
\hline Exchange & 8.35 & 7.67 & 39.52 & 29.15 & 32.43 \\
\hline Induction & -1.34 & -1.54 & -5.84 & -2.93 & -4.76 \\
\hline Dispersion & -5.31 & -3.60 & -35.20 & -28.69 & -29.76 \\
\hline Total & -3.05 & -3.16 & -24.45 & -14.49 & -21.05 \\
\hline Disp/Elst & 1.12 & 0.63 & 1.54 & 2.39 & 1.57 \\
\hline
\end{tabular}


(a)



(b)

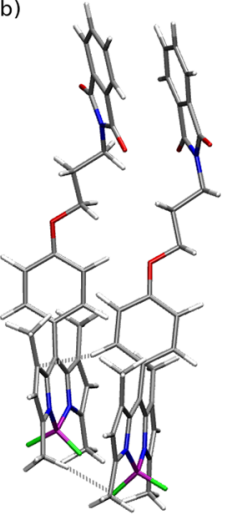

(c)

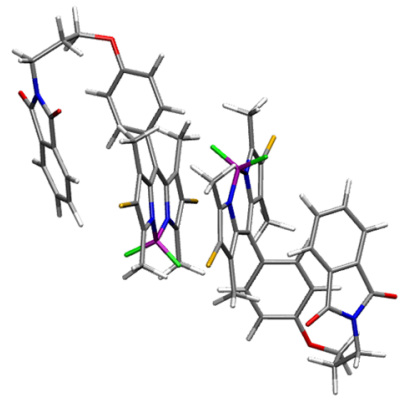

(d)

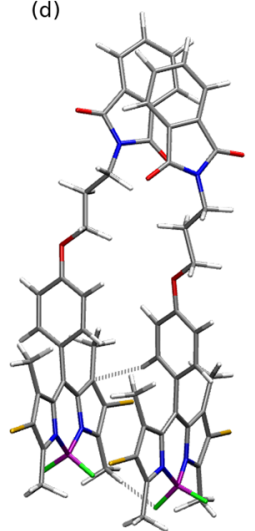

Fig. 5 Vacuum-optimized dimers of 1-H: a bent and $\mathbf{b}$ unfolded and 1-I: $\mathbf{c}$ bent and $\mathbf{d}$ unfolded

in the iodinated species the parallel is favorable with almost zero twist angle, therefore these structures are geometrically not simply comparable.

In each case, the unfolded supermolecular interaction energy as well as the SAPT0 interaction is stronger than the bent one. This arises from the fact that more parts of a molecule can directly interact in the elongated mode than in the bent configuration.

The comparison of the SAPT0 energy decomposition in aug-cc-pVDZ and def2-SVP basis sets performed for the exemplary 1-H bent dimer exhibits the tendencies observed for model 3-H interaction. Namely, the qualitative prediction of all of the interaction energy components with a satisfactory accuracy is possible in the smaller basis set, except the dispersion contribution. It suffers a lot by the truncation of the basis set size and is reproduced only in $75 \%$. This also underestimates significantly the dispersion-to-electrostatic ratio (from 2.52 in aug-cc-pVDZ basis set to 1.87 in def2-SVP) (Table 7). However due to the prohibitively large size of the investigated systems (more than 2500 basis functions), the further results will be reported for def2-SVP basis set and the numerical values of the dispersion component will be given only for the observation of the general tendencies.

One can notice that the nature of the interactions in all of the analyzed dimers is similar to one another. All of these systems are governed by the dispersion interaction (even for def2-SVP underestimated dispersion component). The most pronounced dispersion component is obtained for the 1-H unfolded dimer in which two aromatic parts of the molecule can dispersively attract the other monomer. The comparison of the dispersion energy trends for the 1-H bent and elongated forms confirms the benefit from stacking interactions in these type of systems. This effect is less pronounced in $\mathbf{2 - H}$ and $\mathbf{2}-\mathbf{I}$, where there is only one aromatic core in the molecule and the terminal amino group does not generate much difference upon bending. On the other hand one can notice also slightly more attractive electrostatic components for the elongated structures, for the same structural reasons as for dispersion.

Interaction energy decomposition for the exemplary bent dimer 1-H indicated the strong influence of the dispersion energy component (dispersion-to-electrostatic ratio equal to 2.52 , dispersion-governed interaction). This value is (a)

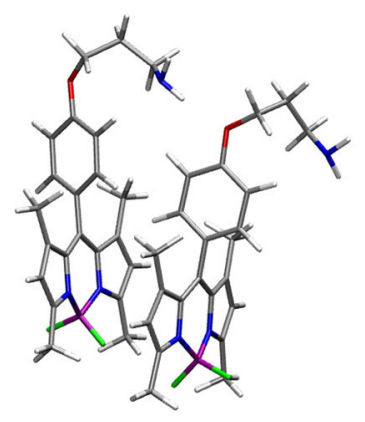

(b)



(c)



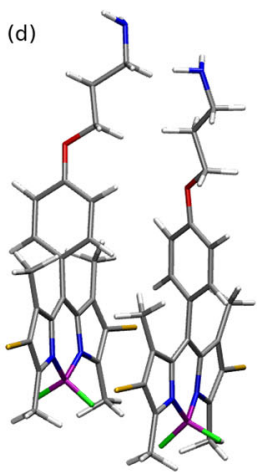

Fig. 6 Vacuum-optimized dimers of 2-H: $\mathbf{a}$ bent and $\mathbf{b}$ unfolded and 2-I: $\mathbf{c}$ bent and $\mathbf{d}$ unfolded 
Table 6 Counterpoise-corrected supermolecular interaction energy $\Delta E_{s m}^{C P}[\mathrm{kcal} / \mathrm{mol}]$ estimated for the fully optimized dimers in vacuum within the $\omega \mathrm{B} 97 \mathrm{X}-\mathrm{D} / \mathrm{def} 2-\mathrm{SVP}$ approach and total SAPT0/def2-SVP interaction energy

\begin{tabular}{llllll}
\hline & \multicolumn{2}{l}{ Supermolecular } & & \multicolumn{2}{l}{ SAPT0 } \\
\cline { 2 - 3 } \cline { 5 - 6 } System & Bent & Unfolded & & Bent & Unfolded \\
\hline $\mathbf{1 - I}$ & -27.47 & -31.88 & & -22.89 & -24.52 \\
$\mathbf{1 - H}$ & -21.31 & -27.03 & & -18.35 & -21.11 \\
$\mathbf{2 - I}$ & -22.99 & -23.57 & & -16.44 & -16.61 \\
$\mathbf{2 - H}$ & -19.97 & -24.16 & & -15.48 & -18.29 \\
\hline
\end{tabular}

similar to the dispersion energy component observed in the prototypical squaraine dimers [41], where for the stacked dimers depending on the mutual monomer displacement it amounts to -46.45 or $-51.51 \mathrm{kcal} / \mathrm{mol}$ for long and short displacement, respectively. Among the other analyzed systems that are important in the context of aggregation, the benzoxathiazole seem to attract dispersively stronger, while the benzooxadiazole exhibit slightly weaker dispersion interaction than $\mathbf{1}-\mathbf{H}$.

\section{Influence of BODIPY dimerization on their photophysical properties}

The intermolecular interactions considered in the present study has mild impact on the vertical absorption spectra of the BODIPY dyes. However, since the experimental measurements of the UV-Vis spectrum of 1-I in the solvent mixture provide the confirmation of the aggregation phenomenon [Rybczynski P, Smolarkiewicz-Wyczachowski A, Ziegler-Borowska M, Bocian S, Piskorz J, KaczmarekKedziera A, Photochemical properties and stability of BODIPY dyes, in preparation], also the influence of the dimerization on electronic excitation is analyzed here.
For the model 3-H crystal-based dimers, the modification of the electronic spectrum is presented in Fig. 7. The increase of the signal intensity can be noticed upon dimerization, however the wavelength remains almost unshifted. Nevertheless, the detailed analysis of the calculated signals demonstrate an interesting tendency, namely for the perpendicular dimers the single absorption band observed in monomer splits to the two component. For the $\mathrm{H}-\mathrm{F}$ interacting perpendicular dimer characterized by the mutual interaction equal to $-3.34 \mathrm{kcal} / \mathrm{mol}$ (SAPT0/aug-cc-pVDZ) this splitting is almost negligible and amounts only to $2 \mathrm{~nm}$, but for the $\mathrm{CH}_{3}-\mathrm{F}$ interacting system with interaction energy equal to $-4.28 \mathrm{kcal} / \mathrm{mol}$, the two lines appear at 407 and $392 \mathrm{~nm}$ (splitting by $15 \mathrm{~nm}$ ). Interestingly, the strongest attraction in parallel dimer, equal to $-13.86 \mathrm{kcal} / \mathrm{mol}$, lead only to the $3 \mathrm{~nm}$ hypsochromic shift of the intensive signal. Apparently here also the splitting is present, however the second line appears blueshifted by $34 \mathrm{~nm}$, yet significantly loosing its intensity. The same set of molecular orbitals is involved in this transition as for the intensive one at $396 \mathrm{~nm}$.

Taking into account the four units from the crystal unit cell, the absorption spectrum also exhibit the splitting with the two intensive transitions placed at 408 and 391 $\mathrm{nm}$ (splitting equal to $17 \mathrm{~nm}$ ). These tendencies remain preserved in different functionals, namely M06-2X, PBE0 and CAM-B3LYP. Panels $b$ and $c$ of Fig. 7 present the main molecular orbitals involved in most intensive transitions in parallel and perpendicular $\mathrm{CH}_{3}-\mathrm{F}$ dimers. It is clear that the excitation in the parallel dimer involves orbital parts localized equally on both units, and in the case of the perpendicular system, the excitation is strongly localized on one monomer only. Thus, each of the transitions observed for the perpendicular dimers (also for the $\mathrm{H}-\mathrm{F}$ one), is degenerated and occurs between two pairs of monomerlocalized orbitals.

Table 7 SAPT0 interaction energy components [kcal/mol] for the 1-H, 1-I, 2-H and 2-I bent and unfolded dimers

\begin{tabular}{|c|c|c|c|c|c|}
\hline Dimer & Electrostatic & Exchange & Induction & Dispersion & $\mathrm{D} / \mathrm{E}$ ratio \\
\hline \multicolumn{6}{|l|}{ aug-cc-pVDZ } \\
\hline $\mathbf{1 - H}$ bent (2298 bf) & -16.18 & 34.47 & -5.09 & -40.77 & 2.52 \\
\hline \multicolumn{6}{|l|}{ def2-SVP } \\
\hline 1-H bent & -16.50 & 31.82 & -4.84 & -30.83 & 1.87 \\
\hline 1-I bent & -20.05 & 40.62 & -5.46 & -38.00 & 1.90 \\
\hline 2-H bent & -13.60 & 33.39 & -5.21 & -30.05 & 2.21 \\
\hline 2-I bent & -14.14 & 39.22 & -4.65 & -36.87 & 2.61 \\
\hline 1-H unfolded & -18.46 & 47.91 & -6.01 & -44.55 & 2.41 \\
\hline 1-I unfolded & -22.17 & 54.55 & -6.55 & -50.35 & 2.27 \\
\hline 2-H unfolded & -16.15 & 37.33 & -5.81 & -33.65 & 2.08 \\
\hline 2-I unfolded & -14.74 & 39.65 & -4.95 & -36.57 & 2.48 \\
\hline
\end{tabular}




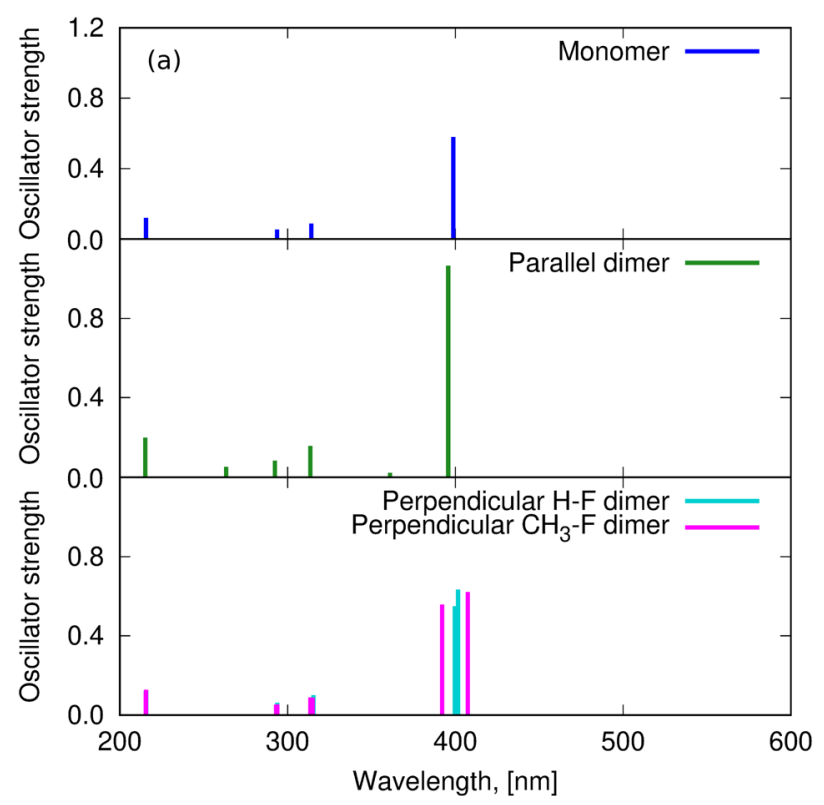

(b)

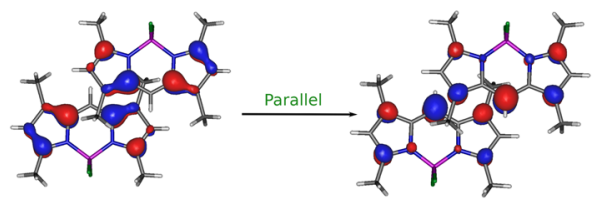

(c)
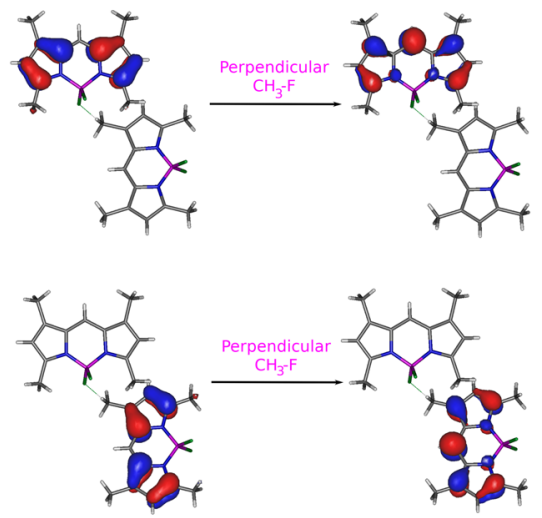

Fig. 7 Absorption spectrum of the crystal dimers of 3-H calculated with the M06-2X/def2-SVP approach in vacuum: a position and intensity of the signals, $\mathbf{b}$ frontier molecular orbitals for the parallel dimer, $\mathbf{c}$ frontier molecular orbitals for the perpendicular $\mathrm{CH}_{3}-\mathrm{F}$ dimer

Similar tendencies are found for the fully optimized 3-H and 3-I dimers and depicted in Fig. 8. Here the antiparallel dimer generates the two spectral lines with the splitting equal to $50 \mathrm{~nm}$ for $\mathbf{3}-\mathbf{H}$ and $45 \mathrm{~nm}$ for 3-I. One should also notice that the size of the splitting is not simply corresponding to the interaction energy changes observed for these dimers in ground state.

The most pronounced modification of the spectrum for model 3-H and 3-I systems can be noticed for the stacked antiparallel dimers, which exhibit the strongest mutual attraction together with the highest value of the dispersion energy component (compare Fig. 8). In this cases the one sharp absorption band with high intensity is splitted into two components of the intensity similar but reduced with respect to the other dimers. The $50 \mathrm{~nm}$ for $\mathbf{3}-\mathbf{H}$ and $45 \mathrm{~nm}$ for 3-I of splitting is noticed and it is the strongest effect observed in the analyzed cases. The parallel dimers of $\mathbf{3}-\mathbf{H}$ and 3-I also exhibit a splitting by 17 to $45 \mathrm{~nm}$ depending on the system, and all of these transitions involve the molecular orbitals delocalized on both monomers. On the other hand, the perpendicular arrangement of the monomers either in 3$\mathbf{H}$ or in 3-I, prohibits the electron density shift between the monomers thus leading to the transitions localized within a single monomer.

The calculated vertical absorption for the 1-H, 1-I, 2$\mathbf{H}$ and 2-I systems is presented in Fig. 9. In all of those cases the dimerization generates the second signal about $400 \mathrm{~nm}$, however only in the case of $\mathbf{1}-\mathbf{H}$ and $\mathbf{2}-\mathbf{H}$ bent dimers the two bands exhibit the comparable intensity.
Thus it can be expected that their presence could be easily noticeable in the experimental spectrum. On the other hand in the remaining cases, the second band shows small intensity thus being covered with the large-intensity signal. The orbitals involved in the most intensive transitions for 1-H and 1-I arise completely from the BODIPY core (compare Fig. S5), thus indicating marginal importance of the side substituent on the system photophysics (what was confirmed form isolated species as well [Rybczynski P, Smolarkiewicz-Wyczachowski A, ZieglerBorowska M, Bocian S, Piskorz J, Kaczmarek-Kedziera A, Photochemical properties and stability of BODIPY dyes, in preparation]). In the case of the bent systems, the orbitals are delocalized on both monomers, but for unfolded, elongated dimers, the electronic excitation occurs in one monomer only (compare molecular orbitals in Fig. S5). The 2-H and 2-I demonstrate even stronger localization of the electron density within the electronic transition to one unit only.

\section{Summary}

The presented study provides the careful analysis of the intermolecular interaction between the BODIPY dyes and its influence on their photophysical properties. The interaction energy decomposition undoubtedly presents the dominating role of the dispersion energy component on the characteristics of the BODIPY dimers, independently on their mutual structural arrangements. The stacking dimers 
Fig. 8 Vertical absorption signals for the optimized dimers of 3-H and 3-I calculated within the M06-2X/def2-SVP approach in vacuum and corresponding frontier orbitals a for 3-H and $\mathbf{b}$ for 3-I

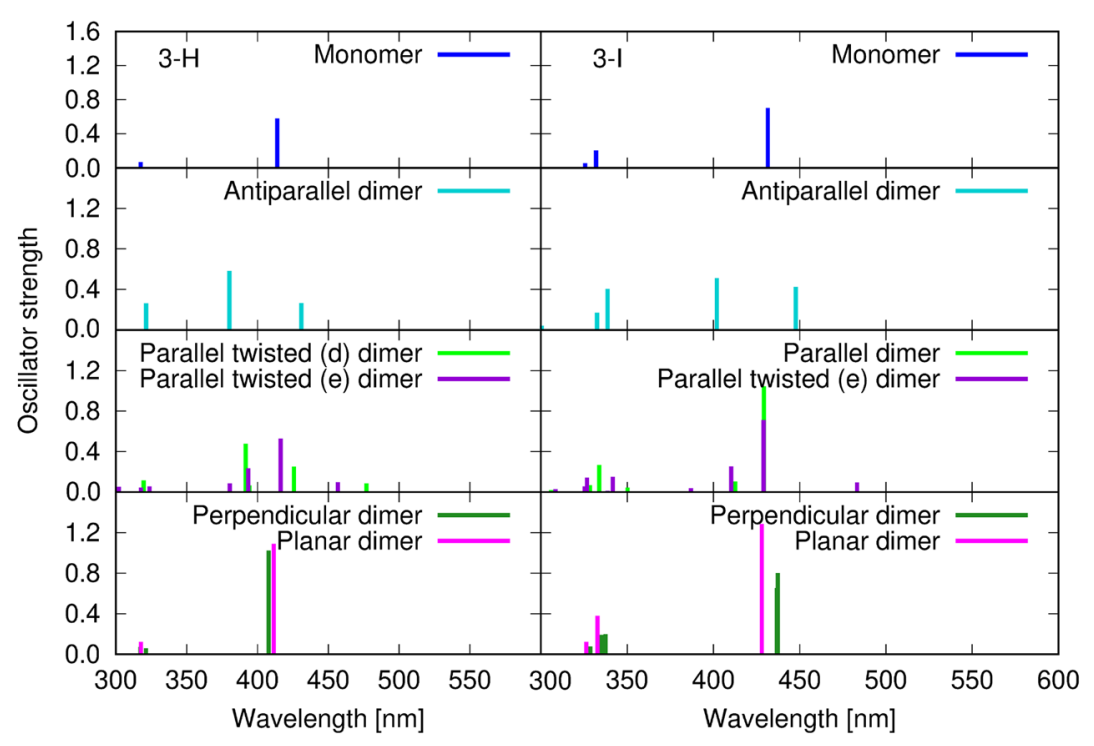

(a)

\section{3-H}


(b)

3-I
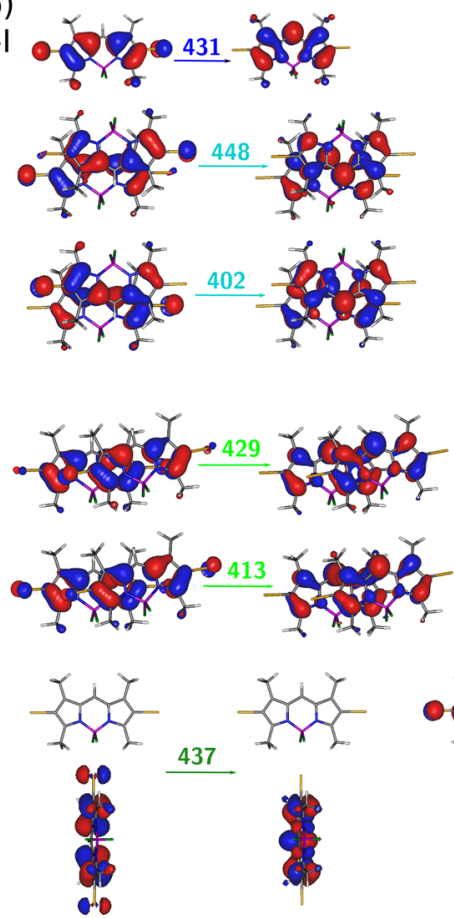

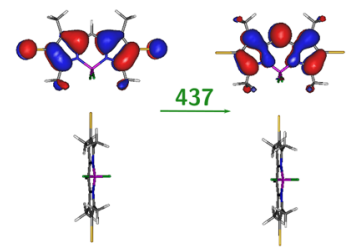

present both in the crystal of model dye and in the antibacterial BODIPYs investigated in the current study present the highest stability of all the analyzed dimers. For the BODIPY dye bearing the large aromatic terminal phthalimide group, the bent monomer preferred in gas phase cannot fully benefit from the mutual stacking interactions between the two dyes forming a dimer, thus leading to the less stable aggregation pattern that its unfolded counterpart. The dimerization of the investigated species causes the modification of the vertical absorption spectra in a manner strongly dependent not solely on the interaction strength but also on the delocalization of the molecular orbitals 
Fig. 9 Absorption spectrum of the bent and unfolded dimers of 1-H, 1-I, 2-H and 2-I calculated with the M06-2X/def2-SVP approach in vacuum (dark lines are for monomers and light lines for dimers)



involved in the transition on both monomers. Therefore, the controlled aggregation of BODIPY dyes can be of practical importance for instance in solid-state applications.

Supplementary Information The online version contains supplementary material available at https://doi.org/10.1007/s11224-021-01759-1.

Acknowledgements Wrocław Center for Networking and Supercomputing is gratefully acknowledged for the generous allotment of computational resources. Authors are indebted to Dr Jarosław Piskorz for the inspiration to the present study.

Author contributions Both authors contributed to the study conception and design. PR provided the experimental basis of the manuscript concept, contributed to the manuscript preparation and participated in data analysis. AKK performed the theoretical calculations, data analysis and contributed to the manuscript preparation.

Availability of data and material The data generated during and/or analyzed during the current study are available from the corresponding author on reasonable request.

\section{Declarations}

Conflict of interest The authors declare that they have no competing interests.

\section{References}

1. Treibs A, Kreuzer FH (1968) Difluorboryl-Komplexe von Di- und Tripyrrylmethenen. Justus Liebigs Ann Chem 718(1):208-223

2. Boens N, Leen V, Dehaen W (2012) Fluorescent indicators based on BODIPY. Chem Soc Rev 41(3):1130-1172

3. Loudet A, Burgess K (2007) BODIPY dyes and their derivatives: Syntheses and spectroscopic properties. Chem Rev 107(11):48914932

4. Guo Z, Park S, Yoon J, Shin I (2014) Recent progress in the development of near-infrared fluorescent probes for bioimaging applications. Chem Soc Rev 43:16-29

5. Zhang XF, Yang X, Niu K, Geng H (2014) Phosphorescence of BODIPY dyes. J Photochem Photobiol A Chem 285:16-20 
6. Gotor R, Ashokkumar P, Hecht M, Keil K, Rurack K (2017) Optical $\mathrm{pH}$ sensor covering the range from $\mathrm{pH}$ 0-14 compatible with mobile-device readout and based on a set of rationally designed indicator dyes. Anal Chem 89(16):8437-8444

7. Yogo T, Urano Y, Ishitsuka Y, Maniwa F, Nagano T (2005) Highly efficient and photostable photosensitizer based on BODIPY chromophore. J Am Chem Soc 127:12162-12163

8. Descalzo AB, Ashokkumar P, Shen Z, Rurack K (2020) On the aggregation behaviour and spectroscopic properties of alkylated and annelated boron-dipyrromethene (BODIPY) dyes in aqueous solution. ChemPhotoChem 4(2):120-131

9. Tokoro Y, Nagai A, Chujo Y (2010) Nanoparticles via Haggregation of amphiphilic BODIPY dyes. Tetrahedron Lett 51(26):3451-3454

10. Prlj A, Vannay L, Corminboeuf C (2017) Fluorescence quenching in BODIPY dyes: The role of intramolecular interactions and charge transfer. Helv Chim Acta 100(6):e1700093

11. Yuan K, Wang X, Mellerup SK, Kozin I, Wang S (2017) SpiroBODIPYs with a diaryl chelate: Impact on aggregation and luminescence. J Org Chem 82(24):13481-13487

12. (2010) Molecular probes handbook, A guide to fluorescent probes and labeling technologies. Life technologies, 11 edition

13. Bergström F, Mikhalyov I, Hägglöf $\mathrm{P}$, Wortmann $\mathrm{R}$, Ny T, Johansson LB- $\AA$ (2002) Dimers of dipyrrometheneboron difluoride (bodipy) with light spectroscopic applications in chemistry and biology. J Am Chem Soc 124:196-202

14. Choi S, Bouffard J, Kim Y (2014) Aggregation-induced emission enhancement of a meso-trifluoromethyl BODIPY via Jaggregation. Chem Sci 5(2):751-755

15. Sampedro A, Ramos-Torres Á, Schwöppe C, Mück-Lichtenfeld C, Helmers I, Bort A, Díaz-Laviada I, Fernández G (2018) Hierarchical self-assembly of BODIPY dyes as a tool to improve the antitumor activity of capsaicin in prostate cancer. Angew Chem Int Ed 57(52):17235-17239

16. Gemen J, Ahrens J, Shimon LJW (2020) R Klajn Modulating the optical properties of bodipy dyes by noncovalent dimerization within a flexible coordination cage. J Am Chem Soc 142:1772117729

17. Kasha M (1963) Energy transfer mechanisms and the molecular exciton model for molecular aggregates. Radiat Res 20:5571

18. Kasha M, Rawls HR, El-Bayoumi MA (1965) The exciton model in molecular spectroscopy. Pure Appl Chem 11:371-392

19. Dziuk B, Ośmiałowski B, Zarychta B, Ejsmont K, Chęcińska L (2019) Symmetric fluoroborate and its boron modification: Crystal and electronic structure. Crystals 9:662

20. Piskorz J, Dlugaszewska J, Porolnik W, Teubert A, Mielcarek J (2020) Boron-dipyrromethene derivatives bearing N-alkyl phthalimide and amine substituents of potential application in the photoinactivation of bacteria. Dyes Pigm 178:108322

21. Schäfer A, Huber C, Ahlrichs R (1994) Fully optimized contracted gaussian basis sets of triple zeta valence quality for atoms li to kr. J Chem Phys 100:5829

22. Schäfer A, Horn H, Ahlrichs R (1992) Fully optimized contracted gaussian basis sets for atoms li to kr. J Chem Phys 97:2571

23. Eichkorn K, Weigend F, Treutler O, Ahlrichs R (1997) Auxiliary basis sets for main row atoms and transition metals and their use to approximate coulomb potentials. Theor Chem Acc 97:119

24. Weigend F, Häser M., Patzelt H, Ahlrichs R (1998) Ri-mp2: optimized auxiliary basis sets and demonstration of efficiency. Chem Phys Lett 294:143
25. Weigend F, Furche F, Ahlrichs R (2003) Gaussian basis sets of quadruple zeta valence quality for atoms $\mathrm{h}-\mathrm{kr}$. J Chem Phys 119:12753

26. Weigend F, Ahlrichs R (2005) Balanced basis sets of split valence, triple zeta valence and quadruple zeta valence quality for $h$ to rn: design and assessment of accuracy. Phys Chem Chem Phys 7:3297-3305

27. Mardirossian N, Head-Gordon M (2017) Thirty years of density functional theory in computational chemistry: an overview and extensive assessment of 200 density functionals. Mol Phys $115: 2315$

28. Wang W, Zhang Y, Wang Y-B. (2014) Noncovalent $\pi \ldots \pi$ interaction between graphene and aromatic molecule: Structure, energy, and nature. J Chem Phys 140:094302

29. Hohenstein EG, Sherrill CD (2012) Wavefunction methods for noncovalent interactions. WIREs Comput Mol Sci 2:304

30. Hohenstein EG, Sherrill CD (2010) Density fitting and cholesky decomposition approximations in symmetry-adapted perturbation theory: Implementation and application to probe the nature of $\pi-\pi$ interactions in linear acenes. J Chem Phys 132:184111

31. Parker TM, Burns LA, Parrish RM, Ryno AG, Sherrill CD (2014) Levels of symmetry adapted perturbation theory (SAPT). I. Efficiency and performance for interaction energies. J Chem Phys 140:094106

32. Zhao Y, Truhlar D (2008) The M06 suite of density functionals for main group thermochemistry, thermochemical kinetics, noncovalent interactions, excited states, and transition elements: two new functionals and systematic testing of four M06-class functionals and 12 other functionals. Theor Chem Acc 120:215-241

33. Isegawa M, Peverati R, Truhlar DG (2012) Performance of recent and high-performance approximate density functionals for timedependent density functional theory calculations of valence and Rydberg electronic transition energies. J Chem Phys 137:244104

34. Azarias C, Habert C, Budzák S, Blase X, Duchemin I, Jacquemin D (2017) Calculations of $n \longrightarrow \pi^{*}$ Transition energies: Comparisons between TD-DFT, ADC, CC, CASPT2, and BSE/GW descriptions. J Phys Chem A 121(32):6122-6134. PMID: 28738157

35. Suellen C, Freitas RG, Loos PF, Jacquemin D, Experiment (2019) Cross-comparisons between TD-DFT, CC, and ADC for transition energies. J Chem Theory Comput 15(8):4581-4590

36. Loos PF, Jacquemin D (2019) Evaluating 0-0 energies with theoretical tools: A short review. ChemPhotoChem 3(9):684-696

37. Blase X, Duchemin I, Jacquemin D (2018) The Bethe-Salpeter equation in chemistry: relations with TD-DFT, applications and challenges. Chem Soc Rev 47:1022-1043

38. Bassal F, Laurent AD, Le Guennic B, Jacquemin D (2017) Exploring the excited-states of squaraine dyes with TD-DFT, SOS-CIS(D) and ADC(2). Dye Pigment 138:169-175

39. Jacquemin D, Duchemin I, Blase X (2017) Is the Bethesalpeter formalism accurate for excitation energies? Comparisons with TD-DFT, CASPT2, and EOM-CCSD. J Phys Chem Lett $8(7): 1524-1529$

40. Xu HJ (2008) 4,4-difluoro-1,3,5,7-tetramethyl-4-bora- 3a,4adiaza-s-indacene. Acta Crystallogr E 64:1672. CCDC deposition number 689162

41. Kaczmarek-Kędziera A, Żuchowski PS, Kȩdziera D (2020) Nature of intermolecular interaction in squaraine dimers. Sci Rep 10:19670

Publisher's note Springer Nature remains neutral with regard to jurisdictional claims in published maps and institutional affiliations. 